\title{
Hongos asociados a Echinochloa colona en zonas de producción de arroz en Paraguay
}

\section{Fungi associated to Echinochloa colona in rice production áreas in Paraguay}

\author{
Lidia Quintana ${ }^{1}$, Susana Gutiérrez ${ }^{2}$, Nilsa Sotomayor ${ }^{1}$, Raquel Vigo ${ }^{1}$ \\ 1 Universidad Nacional de Itapúa, Facultad de Ciencias Agropecuarias. Encarnación, \\ Paraguay. \\ 2 Universidad Nacional del Nordeste, Facultad de Ciencias Agrarias. Corrientes, \\ Argentina. \\ Autor de correspondencia: lviedmaq@gmail.com
}

\begin{abstract}
Resumen: Las especies Echinochloa colona, E. crusgalli y E. oryzicola se encuentran entre los grupos más importantes de malezas en los arrozales. Estas malezas además de causar reducción del rendimiento pueden hospedar a hongos fitopatógenos para el cultivo del arroz. El trabajo de investigación se llevó a cabo durante el ciclo del cultivo de arroz 2015/2016 con la colecta al azar de 150 muestras de la maleza de arroz Echinochloa colona en campos de cultivo de arroz. El objetivo del trabajo fue identificar y caracterizar especies fúngicas asociadas a la maleza Echinochloa colona. Se utilizaron técnicas de rutina para el estudio de enfermedades de plantas como cámaras húmedas, siembras y aislamientos in vitro y estudios morfométricos de microorganismos. Como resultados de estas actividades, se identificaron los siguientes hongos asociados a dicha maleza: Curvularia lunata, Bipolaris oryzae, Alternaria padwickii y Alternaria sp. La especie C. lunata registró la mayor incidencia $(92 \%)$.
\end{abstract}

Palabras clave: Oryza sativa, maleza, Echinochloa, hongos.

Abstract: E. colona, E. crusgalli and E.oryzicola are among the most important groups of weeds in paddy fields. These weeds, in addition to causing a reduction in yield, can host phytopathogenic fungi for rice crop. The research work was carried out during the 2015/2016 rice cycle with the aim of identify and characterize fungal species associated with the rice weed. 150 samples of Echinochloa colona plants were randomly collected in commercial rice fields. Routine techniques was used for the study of plant diseases such as humid chambers, in vitro cultures, isolations, and morphometric studies of microorganisms. As a result of these activities, were indentified 4 fungi genera associated with the mentioned weed: Curvularia lunata, Bipolaris oryzae. Alternaria padwickii y Alternaria spp. C.lunata register the higher incidence (92\%).

Keywords: Oryza sativa, weed, Echinochloa, fungi. 


\section{INTRODUCCIÓN}

El cultivo del arroz con riego ha experimentado un crecimiento y expansión muy rápida, en el Paraguay, debido al uso de mejores técnicas de cultivo. La superficie sembrada en el ciclo $2019 / 2020$ asciende a $184.000 \mathrm{ha}^{(1)}$. En el país se produce aproximadamente unas 897.000 ton de arroz al año(2) las cuales aproximadamente el $30 \%$ se utiliza para el consumo nacional y el resto es exportado a terceros países como Brasil, Chile, Perú, entre otros.

Uno de los factores más limitantes en la producción de arroz, lo constituyen las malezas, ya que causan daños directos e indirectos al cultivo por la competencia de luz, agua y nutrientes. Asimismo, pueden ser hospederas de enfermedades que afectan el crecimiento normal del cultivo ${ }^{(3,4)}$.

Las principales malezas que se encuentran en los arrozales incluyen las siguientes especies Ageratum conyzoides, Cyperus difformis, Cyperus iria, Echinochloa colona, Echinochloa crusgalli, Echinochloa oryzicola, Fimbristylis miliacea, Ischaemum rugosum, Monochoria vaginalis, Oryza sativa (arroz

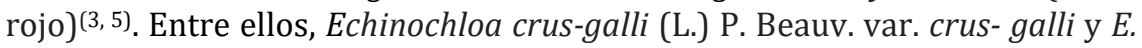
colona (L.) Link son consideradas las especies de malezas más importantes y temibles del cultivo del arroz por su alta capacidad de distribución de semilla [6]. Las mencionadas malezas asimismo son hospederas de varios patógenos causantes de enfermedades del arroz $(3,7)$.

En Paraguay, entre las malezas gramíneas que causan pérdidas más importantes a cultivos de arroz se encuentran E.colona y el arroz colorado existiendo varias áreas muy infestadas o contaminadas, convirtiéndose en importante amenaza a la producción de arroz, debido a que no se cuenta con un programa de certificación de semilla ${ }^{(8)}$. Sin embargo, otro aspecto a considerar sobre estas malezas, es su rol como hospedantes de microorganismos que ocasionan enfermedades en los cultivos. Al respecto, en lo que se refiere al cultivo de arroz, la bibliografía menciona a especies de Echinochloa como hospedante de numerosos géneros y/o especies de hongos que afectan al cultivo de arroz, tales como Pyricularia sp, Helminthosporium oryzae, H. sativum, H. australiensis, Exserohilum spp, Drechslera rostrata, Curvularia lunata y C. geniculata $(9,10,11,7)$.

Considerando la presencia importante que representa el género Echinochloa, como maleza acompañando al cultivo de arroz en el Paraguay, se realizó este trabajo a fin de identificar y caracterizar hongos fitopatógenos del arroz que puedan estar asociados a plantas de E. colona. 


\section{MATERIALES Y MÉTODOS}

La investigación se realizó en el laboratorio de Microbiología, de la Facultad de Ciencias y Tecnología Universidad Nacional de Itapúa, (UNI), Encarnación, en el ciclo del cultivo 2015/2016. Se recolectaron 150 muestras de plantas de la maleza Echinochloa colona que presentaba síntomas de manchas foliares en campos comerciales de cultivos de arroz, localizados en los departamentos de Misiones, Itapúa, y Caazapá; dichas muestras fueron analizadas mediante la preparación de cámaras húmedas con segmentos de tejidos foliares sintomáticos enfermos. Posteriormente se realizaron siembras in vitro de este material en medio de cultivo con Agar papa glucosado 1,5\%, a pH 6, incubados en condiciones de $12 \mathrm{~h} \mathrm{luz}, 12 \mathrm{~h}$ oscuridad, a partir de los cuales desarrollaron colonias fúngicas de diversos microorganismos. La identificación de los mismos se realizó analizando características culturales y morfométricas y utilizando la bibliografía específica(12, 13, 14). También se determinó el porcentaje de frecuencia e incidencia de cada uno de los microorganismos identificados.

Los datos fueron analizados utilizando tablas de frecuencia absoluta y porcentuales, que indican el número de observaciones de cada categoría separando las especies fúngicas por su mayor o menor frecuencia.

\section{RESULTADOS Y DISCUSIÓN}

La frecuencia de especies fúngicas identificadas en la maleza E.colona se presenta en la siguiente Tabla 1:

Tabla 1. Especies fúngicas provenientes de la maleza gramínea E.colona

\begin{tabular}{lll}
\hline Géneros/especie & Frecuencia total & Incidencia (\%) \\
\hline Alternaria padwickii & 30 & 3 \\
Alternaria sp. & 120 & 13 \\
Bipolaris oryzae & 104 & 12 \\
Curvularia lunata & 825 & 92 \\
Total: 1079 & & \\
Media: 269,7 & & \\
Moda: Curvularia lunata & \\
\hline
\end{tabular}

Durante la campaña agrícola 2015-2016, en muestras de plantas de E. colona con síntomas foliares, se identificaron los siguientes microorganismos: Curvularia lunata, Bipolaris oryzae, Alternaria padwickii y Alternaria spp. Todos éstos hongos también pueden parasitar a diferentes órganos de la 
planta de arroz (hojas, vainas foliares, panojas, semillas), causando una gran variedad de sintomatologías $(7,15,10,13,14)$.

Con respecto a los géneros Curvularia y Bipolaris, corresponde a hongos presentes en semillas, que por lo general afectan a especies gramíneas (Avena, Brachiaria, Bromus, Cynodon, Digitaria, Echinocloa, Hordeum, Oryza, entre otras), causando manchas y tizones en hojas ${ }^{(16,17)}$. Aunque también pueden afectar a plántulas de estas especies de plantas. De igual manera, $A$. padwickii y Alternaria spp son organismos presentes en semillas, causantes de muerte de plántulas, manchas foliares y tizones en cultivos de arroz y diferentes especies de Echinochloa ${ }^{(15)}$.

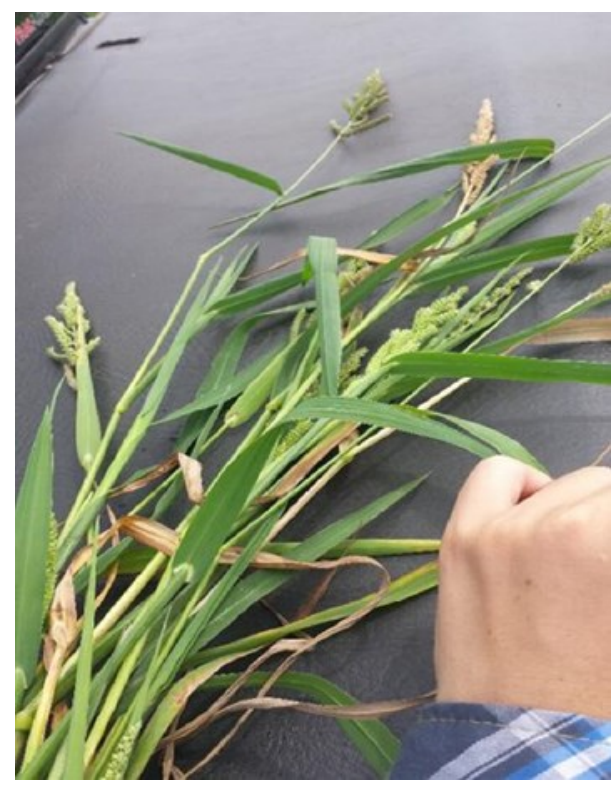

Figura1: Hojas afectadas en E.colona.

Los síntomas foliares observados en el campo en plantas de E. colona se describen como pequeñas manchas cloróticas que posteriormente se vuelven necróticas y evolucionan causando necrosis en la mayor parte de la superficie foliar (Figura 1). Las características morfológicas de los hongos asociados a la mencionada maleza se describen a continuación:

Alternaria padwickii presenta conidios que crecen solos en conidióforos cortos hialinos emergiendo directamente del sustrato o en conidióforos desde un micelio suave, esponjoso de color blanco grisáceo. Los conidios al inicio son sub-hialinos y más tarde color paja a marrón más oscuro que el micelio con un prominente apéndice. Observaciones microscópicas (400x) 
muestran conidio de 3 a 5 septas, a menudo con constricción en la célula basal y pico largo con medidas 95-160 $\mu \mathrm{m} \times 11-30 \times 11-20 \mu \mathrm{m}^{(12,13)}$ (Ver Figura 2).

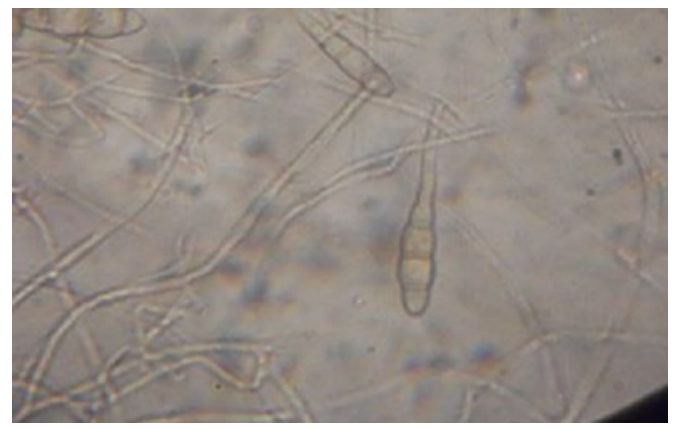

Figura 2: Conidios de Alternaria padwickii.

Alternaria spp presenta los conidióforos simples, tabicados, de forma alargada u ovoide. En el extremo del conidióforo se forman unos conidios de color pardo, con septos transversales y verticales de disposición irregular(16) (Ver Figura 3).

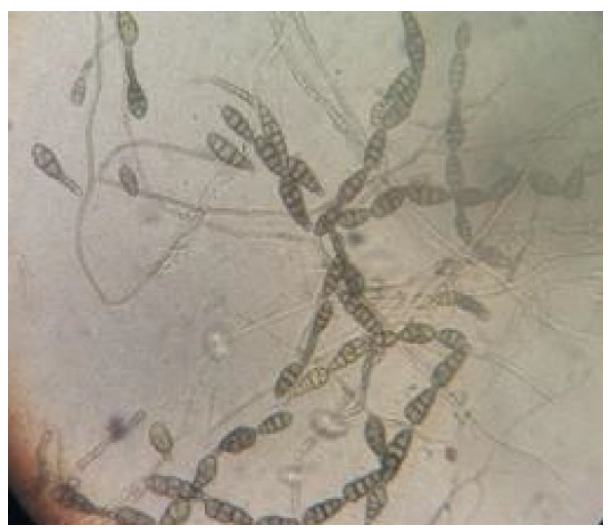

Figura3: Conidios de Alternaria spp.

Curvularia lunata presenta micelios aéreos escasos o ausentes; de color marrón claro a marrón oscuro con abundante ramificación. Los conidióforos son solitarios o en grupos; color marrón oscuro; recto, simple; surgiendo directamente del sustrato. Los conidios son elipsoidales, a menudo curvados o semilunar, redondeados en los extremos, se estrecha ligeramente hacia la base, marrón pálido a marrón oscuro, septos generalmente 3-5 con medidas de 16,30-24,75 × 7,00-13,0 Las colonias son zonadas y fieltradas; el área 
conidial es de color gris verdoso con un margen grisáceo. La apariencia de la colonia en el reverso de la placa de agar es zonada y gris(12) (ver Figura 4).

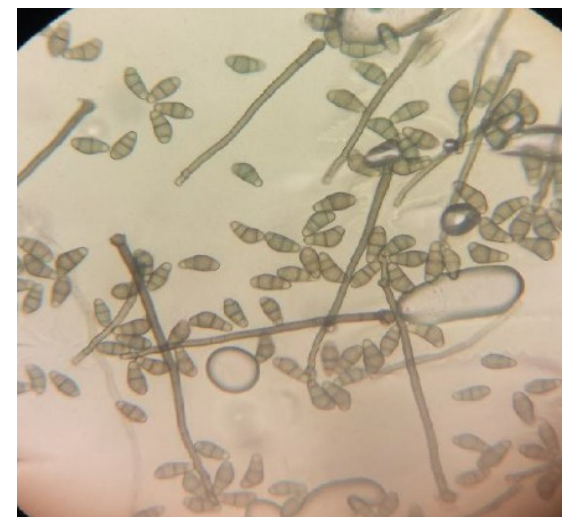

Figura 4: Conidios de Curvularia lunata.

Bipolaris oryzae presenta micelio gris a castaño oscuro con conidióforos septados, emergiendo solos o en pequeños grupos, conidios ligeramente recto o curvos, en la mayoría de los casos curvos de color castaño oscuro a oliváceo con 6-13 septos en células transversales con medidas de 39-100 × $12.0 \mu \mathrm{m}(12)$, Figura 5. Las colonias en PDA crecen, vellosas en el centro, se vuelven algodonosas hacia el margen, gris amarillento en el centro y grises hacia el margen. La colonia aparece negra en el reverso de la placa de agar(12, 13), (ver Figura 5).

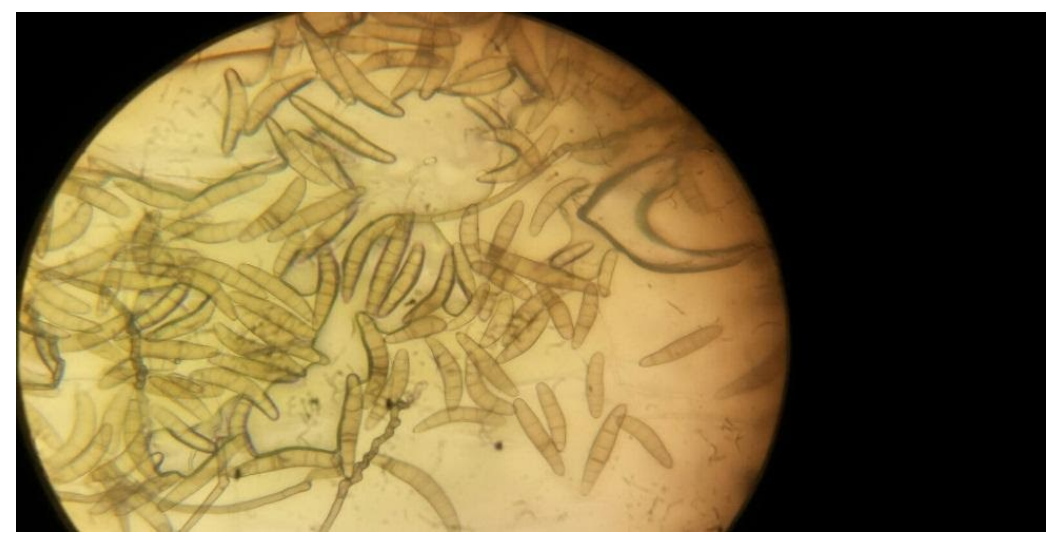

Figura 5: Conidios de Bipolaris oryzae.

Cabe resaltar la importancia que representan la presencia de éstos organismos sobre especies de Echinochloa con efecto de micoherbicidas para 
el control de malezas. Al respecto, se obtuvieron resultados positivos de control, principalmente con Curvularia lunata y Alternaria pellucida ${ }^{(11,18)}$.

\section{CONCLUSIÓN}

Se identificaron 4 géneros de hongos asociados a la maleza de arroz Echinochloa colona: Alternaria padwickii, Alternaria spp, Bipolaris oryzae y Curvularia.lunata. La especie C. lunata registró la mayor incidencia (92\%) en las muestras analizadas.

\section{AGRADECIMIENTOS}

A PROCIENCIA-CONACYT por la financiación de esta investigación.

\section{REFERENCIAS BIBLIGRÁFICAS}

1. INBIO (Instituto de Biotecnología Agrícola). Estimación de la superficie cultivos de verano soja-arroz 2019/2020 y otros [Internet]. 2019 [20 marzo 2020]. Disponible en: http://www.inbio.org.py/wp.content/upload/mapas/soja-arroz-maiz2019-2020

2. FAOSTAT. Seguimiento del mercado del arroz [Internet]. 2018 [Consultado 20 marzo 2020]; Disponible en: http://www.fao.org/economic/est/publicaciones/publicaciones-sobreel-arroz/seguimiento-del-mercado-

3. Suárez L, Alzalone A, Moreno 0. 2004. Evaluación del herbicida halosulfuron - metil para el control de malezas en el cultivo de arroz (Oryza sativa L.). Bioagro [Internet]. 2004 [consultado 15 marzo 2020]; (3):173-182.

Disponible

en: https://www.researchgate.net/profile/Anzalone_Alvaro/publication/28 086573_Evaluacion_del_herbicida_halosulfuronmetil_para_el_control_de_malezas_en_el_cultivo_de_arroz_Oryza_sativa_L /links/00463525d46d6cb0bd000000/Evaluacion-del-herbicidahalosulfuron-metil-para-el-control-de-malezas-en-el-cultivo-de-arrozOryza-sativa-L.pdf

4. Zhang WM, Moody K. Watson K. Responses of Echinochloa species and rice (Oryza sativa) to indigenous pathogenis fungi. Plant dis. [Internet] 2006 [Consultado 5 de Mayo 2020]; (80)1053-1058. Disponible en: https://www.apsnet.org/publications/plantdisease/backissues/Docum ents/1996Articles/PlantDisease80n09_1053.PDF

5. Moreira EJ, Nippes,H. Manual de Identificación de plantas infestantes: arroz. Sao Paulo, FMC Agricultural Products. 2010;854 p. 
6. Holm LG, Plucknett DL, Pancho JV, Herberger JP. The Word's Worst Weeds. Distribution and Biology. Honolulu: The University Press of Hawaii; 1977.

7. Bastida L, Gutiérrez SA, Carmona MA. Aislamiento y caracterización sintomática de Pyricularia sp en arroz y otros hospedantes en la provincia de Corrientes (Arg.). Summa Phytop. 2019;45(2):200-203.

8. Vigo R, Sotomayor N. Malezas gramíneas como hospederos de hongos asociados al arroz en regiones productoras de arroz. [Tesis de Maestría en Protección de Cultivos]. Facultad de Ciencias Agropecuarias y Forestales. Universidad Nacional de Itapúa, Encarnación; 2016.

9. Serghat S, Mradmi K, Ouazzani Touhami A, Douira A. Rice Leaf Pathogenic Fungi on Wheath, Oat, Echinochloa phyllopogon and Phragmites australis. Phytopathol Mediterr [Internet]. 2005 [Consultado 12 de marzo 2020]; (44):44-49.

Disponible

en: https://pdfs.semanticscholar.org/e321/4b115b785b6db1159fe392820 a56ace68d6d.pdf

10. Kuruppiah R, Seetharaman K. Studies on the mycoflora associated with Colona- Echinochloa - a worst weed in rice systems, J Ecobio [Internet] 2006; [consultado 16 de Agosto 2019]. Disponible en: https://www.cabi.org

11. Motlagh MRS. Reaction of major weeds and some rice cultivars to Alternaria pellucida- a potential biocontrol agent. AJCS [Internet] 2010 [Consultado 5 de Mayo 2020]; 4(6):457. Disponible en: http://www.cropj.com/motlagh_4_6_2010_457_460.pdf

12. Ellis MB. Dematiaceous Hyphomycetes. 3 ed. Surrey, CAB (UK) 1993; 608 p.

13. Mew TW, Gonzales P. A handbook of rice seed borne fungi. Los Baños (Philippines): International Rice Research Institute and Enfield. N.H. (USA); Science Publishers, Inc. 2002; 83 p.

14. Webster RK, Gunnell, PS. Compendium of Rice Diseases. St Paul, MN; APS Press; 1992.62 p.

15. Jyothi G, Vijayavani S, Reddy RK, Sreenivas V. Pathogenicity of Fusarium oxysporum and Curvularia lunata as a mycoherbicide for the control of Echinochloa crusgalli (Barnyard grass). JBiopest [Internet]. 2010 [consultado 2019 Ag. 15]; 3 (3):559-562. Disponible en: http://www.jebas.org/wp-content/uploads/2014/09/Jyothi-et-alJEBAS.pdf

16. Ou SH. Rice Diseases. CMI (UK); 1972, 368 p.

17. Barnett HL, Hunter BB. Illustrated genera imperfect fungi. 4 ed. SP (MN): APS Press; 1998; 218 p. 
18. Motlagh MRS. Isolation and characterization of some important fungi from Echinochloa sp. the potential agents to control rice weeds. POJ [Internet]. 2011 [consultado 5 de Mayo 2020]; 2011;4(3):163. Disponible en: https://pdfs.semanticscholar.org/3963/e183ed86601f9b5e261d8ef4b6 171a7045db.pdf

19. Piontelli EL, Grixolli MA. Especies graminícolas de Bipolaris, Drechslera y Curvularia en la V Región(Chile): aspectos morfológicos y biogeográficos. Boletin Micológico. 1994;9(1-2):87-97.

20. Marin FY, Senwanna C, Cheewangkoon R, Crous PW. New species and records of Bipolaris and Curvularia from Thailand. Mycosphere. 2017;(9):1556-1574. 2017. 